\title{
What Keeps Patients from Adhering to a Home Blood Pressure Program?
}

\author{
Laura S. Huff, MD, Linda Zittleman, MSPH, Lauren DeAlleaume, MD, \\ Jackie Bernstein, MPH, Robert Chavez, BS, Christin Sutter, BS, \\ William G. LeBlanc, PhD, and Bennett Parnes, MD
}

Background: Home blood pressure monitoring (HBPM) predicts cardiovascular risk and increases hypertension control. Non-participation in HBPM is prevalent and decreases the potential benefit.

Methods: Telephone surveys were conducted with a random quota sample of non-participants in a HBPM program, which supplied a complimentary automated blood pressure cuff and utilized a centralized reporting system. Questioning assessed use of monitors, perceived benefit, communication with providers, and barriers.

Results: There were 320 completed surveys (response rate 53\%). of non-participants, $70.2 \%$ still used HBPM cuffs and $58 \%$ communicated values to providers. Spanish-speakers were 4.4 times more likely to not use cuffs $(95 \% \mathrm{CI}, 2.22-8.885)$. Barriers to participation were largely personal (forgetting, not having time, or self-described laziness). Reasons for not communicating readings with providers were largely clinic factors (no doctor visit, doctor didn't ask, thinking doctor wouldn't care). Lack of knowledge of HBPM and program design also contributed. After being surveyed, patients were over three times more likely to use the central reporting system.

Discussion: Most non-participants still used HBPM and communicated values to providers, suggesting many "drop-outs" may still receive clinical benefit. However, much valuable information is not utilized. Future programs should focus on reminder systems, patient motivation, education, and minimizing time involvement.

Keywords: Home Blood Pressure Monitoring, Hypertension, Hypertension Control, Nonparticipation, Self-management

Hypertension is the leading cause of cardiovascular mortality and morbidity in the United States and worldwide. ${ }^{1}$ In $2007,29 \%$ of all US adults 18 years and older were hypertensive. ${ }^{2}$ Elevated blood pressure (BP) accounts for $27 \%$ of all cardiovascular disease events among women, $37 \%$ of all cardiovascular disease events among men, ${ }^{3}$ and $56 \%$ of chronic kidney disease in the United States. ${ }^{4}$

This article was externally peer reviewed.

From the Department of Family Medicine, University of Colorado, Denver.

Funding: This project was funded by a Cancer, Cardiovascular Disease, and Pulmonary Disease grant and a student research grant at the University of Colorado Denver, School of Medicine.

Conflict of interest: none declared.

Corresponding author: Laura Huff, 833 Dexter Street \#207, Denver, CO 80220, (E-mail: LJSturgess@gmail.com, Laura. Huff@ucdenver.edu.
Despite this, BP control is inadequate in the United States. ${ }^{5}$ In 2007, 50.1\% of patients with hypertension had controlled BP $(<140 \mathrm{~mm} \mathrm{Hg}$ systolic and $<90 \mathrm{~mm} \mathrm{Hg}$ diastolic), ${ }^{2}$ an improvement over 1999 to 2000 , when only $29 \%$ of all patients with hypertension were in control. ${ }^{2,5}$ Home blood pressure monitoring (HBPM) is growing in popularity among patients and providers as a strategy to improve hypertension control. ${ }^{6,7}$ Patients more likely to monitor home blood pressures (HBPs) recall a doctor's recommendation to do so; are older than 65; have a history of stroke or transient ischemic attack; and have higher levels of hypertension knowledge, education level, or socioeconomic status. ${ }^{8-10}$

In 2008, the American Heart Association (AHA) and others published the "Call to Action on Use and Reimbursement for Home BP Monitoring," which states that "HBPM should become a routine 
component of BP measurement in the majority of patients with hypertension." HBP correlates strongly with 24-hour ambulatory monitoring and predicts target organ damage and cardiovascular risk better than office BP (class IIa; level of evidence, A). ${ }^{11}$

Evidence that home monitoring improves overall $\mathrm{BP}$ control is increasing, especially when data are communicated to health care providers on a regular basis. ${ }^{11} \mathrm{~A}$ meta-analysis in 2006 found HBPM was associated with a reduction in BP. Additional interventions further improved BP control, such as a system to review home readings and a reminder system. ${ }^{12}$ Even in well designed systems, patient participation may be low. One Estonian study implemented HBPM for 1 year and experienced an initial adherence to daily HBPM of $89 \%$. After 2 months, participation declined to $64 \%$ and remained stable for the remainder of the year. Nonadherence was mainly because of work environment, such as night shifts and travel. Early dropout was primarily because of changing residence. After the 1-year trial, only half of the study population were willing to continue HBPM. ${ }^{13}$ This descriptive study evaluated the utility and validity of HBPM and did not describe barriers to participation.

Nonparticipation is not unique to HBPM. It is well-described in other self-management programs, predominantly for diabetes. In one randomized controlled trial comparing a self-management intervention to control, $25 \%$ of consented patients (30 of 119) dropped out of the intervention group before the intervention began, and only $66 \%$ completed the 1-year intervention. Drop out was largely because of practical or personal factors and was significantly associated with lower education level. In general, dropouts did not take their disease less seriously or express lower levels of self-management. $^{14}$

Using focus groups, another study of economically disadvantaged patients identified several barriers to diabetes self-management: individual barriers (emotional toll of disease, stress, frustration, social isolation, interpersonal conflicts, depression, fear, and denial); educational barriers; and system barriers (need for follow-up and refresher courses, support groups, nutrition and medication education in different modalities, and expanded clinic hours). ${ }^{15}$
Although extrapolations can be made from existing literature on nonparticipation in self-management, there has never been a study evaluating nonparticipation in HBPM programs in the United States. Furthermore, whether patients who stop participating in formal programs continue to use their HBP monitors and thus benefit from monitoring also remains unclear. Exploration in this area will identify barriers as well as areas for improvement so that future HBPM programs can have a stronger impact on the reduction of cardiovascular risk.

\section{Methods \\ Context}

Achieving Cardiovascular Excellence in Colorado (A CARE) is an HBPM program based at the University of Colorado, Denver, Department of Family Medicine. Patients were enrolled in the program from 26 geographically and ethnically diverse primary care practices within the State Network of Colorado Practices and Partnerships research network. Adults older than 18 years with 2 or more elevated $\mathrm{BP}$ readings, a history of hypertension, or who are currently being treated for hypertension were eligible to receive a free HBP cuff (model DS-1902PV, Prevention, Nissei, Ltd in Indonesia). This cuff complied with current industry standards by having automatic inflation, oscillometric detection, and a memory feature that calculated averages. ${ }^{16}$ Wrist cuffs (model WS-820, Prevention) were provided for those patients whose arm circumference was too large for the arm cuff $(>17$ inches). Both the arm and wrist cuffs have been validated using standard international protocols as recommended by the AHA. ${ }^{11}$

Each enrollee watched a short training video to learn correct BP cuff technique, which was verified by a medical assistant or nurse. Patients were asked to check their HBP daily (varying time of day) and to report the average HBP through an automated reporting system (via internet or telephone) 1 week after enrollment and then monthly. Patients were instructed to report values whether they were high, low, or normal. Patients received automated phone calls or E-mail reminders to report, as well as a reminder if no report was received after the due date. All patient components were available in English and Spanish.

HBP feedback reports were sent to patients and providers. Patient feedback reports included a 
graph of HBP in relation to the target HBP and a graph of HBP over time. Clinically oriented provider feedback reports with HBPM results were sent weekly. These provider reports also contained a list of enrollees who had not reported during the preceding 60 days. At the time of this assessment, more than 2800 patients were enrolled in A CARE, of which more than half were not using the reporting system.

\section{Design}

This quality improvement project served 3 purposes among nonusers of the reporting system: (1) to measure cuff use, (2) to identify barriers to participation, and (3) to increase reporting through motivational patient education. Of 26 clinics, 22 participated in this evaluation. The 4 nonparticipating clinics included 2 with insufficient numbers of enrollees, one that dropped out of A CARE, and one that elected to not participate. A 20-question telephone survey was developed with input from the participating clinics. Qualitative and quantitative questioning assessed patient use of home monitors, perspectives on HBP usefulness, barriers to HBPM, and communication with providers. Surveys were translated and conducted in English and Spanish.

Patients were identified as nonreporters if they had never used the central reporting system (never reporters) or had not reported in the preceding 90 days (inactive reporters). Nonreporters were eligible if they had received an HBP cuff, continued to receive care at an A CARE clinic, had a valid phone number in their clinic's database, and could complete a telephone interview. Eligible patients were divided into 3 categories: "never reporters," "inactive reporters with controlled hypertension," and "inactive reporters with uncontrolled hypertension." Controlled or uncontrolled hypertension was determined based on the patient's last HBP report.

Eligible patients were assigned a randomly generated number and called sequentially until a preestablished quota of interviews was completed or call lists at each clinic were exhausted. Multiple contact attempts were made, including morning, afternoon, and evening calls. Survey quotas were set at $20 \%$ of the "never reporter" group and $20 \%$ of the "inactive reporter" group at each clinic. Among "inactive reporters," more "inactive reporters with uncontrolled hypertension" were surveyed than "inactive reporters with controlled hypertension" (3:1) because of the increased clinical value of understanding patients with uncontrolled hypertension (Figure 1). In 9 smaller clinics, the 20\% quota was increased to yield a minimum of 10 surveys per clinic to gather sufficient clinic-level data.

\section{Analyses}

De-identified data were aggregated and provided to the evaluation team. Data were double entered and cross-checked to identify and correct errors. This research was approved by the Colorado Multiple Institutional Review Board.

$\chi^{2}$ measures of association were calculated to determine if there was an association among the demographic characteristics of sex, race/ethnicity, or diabetic status and response status of the surveyed nonreporters. $\chi^{2}$ analysis also was used to measure association between survey status and reporting status after the survey. An independent samples $t$ test was performed to determine if there was an age difference between nonreporters who were surveyed not surveyed. A logistic regression analysis was used to evaluate the multivariate influence of age, language, sex, diabetic status, urban/ rural setting, and reporter status (never reporter, inactive reporter with controlled hypertension, or inactive reporter with uncontrolled hypertension) on the outcome of using the HBP cuff. All analyses were performed using SAS software (version 9.2, SAS, Inc., Cary, NC).

Figure 1. Survey participant categories.

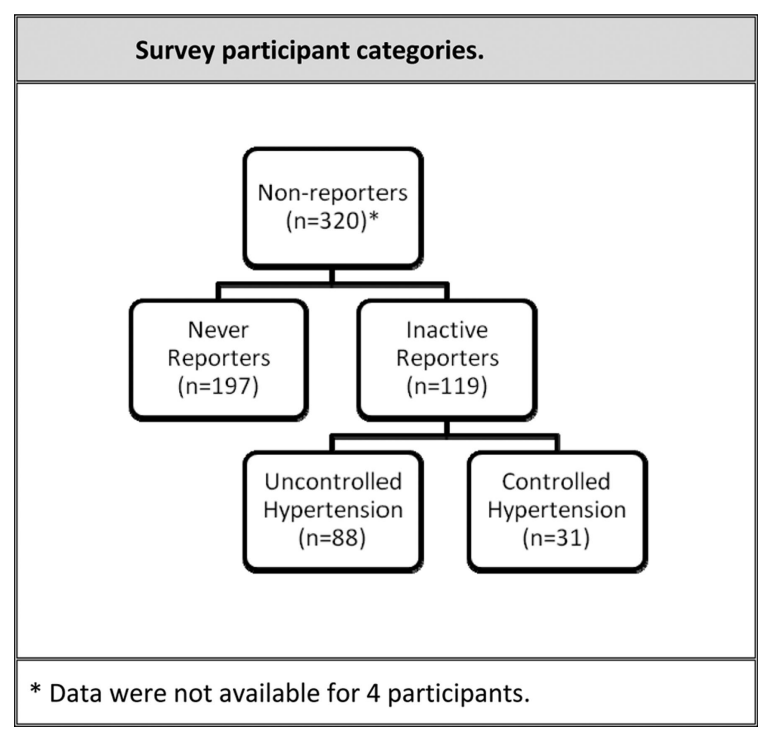


Qualitative data were collected using a structured interview guide that asked: (1) What has kept you from checking your blood pressure regularly at home? (2) What are some of the reasons why you have not reported/stopped reporting your blood pressure? (3) What are some reasons you do not share your blood pressure readings with your health care provider? Multiple responses were allowed. Interviews were transcribed, and an interactive process was used to analyze the data and identify emerging themes. Responses were initially coded using an a priori code list of reasons for nonparticipation that was created based on the literature and anecdotal reports. Unique responses were coded separately to be reviewed by an analyst, generating an expanded, revised code list to develop a set of emergent, provisional themes. The complete set of coded data were reviewed by 3 authors (LH, LZ, BP) to develop a final set of themes that described the common reasons for nonparticipation: personal factors, clinic factors, HBPM program system factors, cuff factors, lack of knowledge about $\mathrm{BP}$ and the HBPM program, or other.

\section{Results}

Calls to 603 eligible patients were attempted to reach the quota of 320 surveys, yielding a response rate of $53 \%$. Among the $47 \%$ who did not complete the interview, up to 9 calls were made trying to reach the participant. Those who did not complete interviews were primarily patients who did not answer their phones or who were not available. Only 17 patients refused to be surveyed. Call lists were not exhausted in most clinics because the quota was met before some of those on the lists were ever called. However, at a few clinics the call lists were exhausted before reaching the clinic's quota. These instances resulted in completing 13 fewer surveys than the quota for all of these clinics combined. Oversampling of small clinics yielded an additional 22 surveys. Of the 320 surveys, 2 surveys were not included in the analysis because the participants did not meet eligibility criteria. Two additional surveys were not fully completed. Therefore, this analysis included a total of 318 surveys, of which 316 were complete.

The surveyed group had a mean age that was 4 years older $(P<.0001)$ and had more women $(P=.0211)$ than nonreporters who were not surveyed. Diabetes status and race were not statistically different. Practice location (rural/urban), preferred language (Spanish/English), and previous reporting data were not available for nonsurveyed nonreporters, which prohibited comparative analysis of these characteristics. Survey participant demographics are shown in Figure 2.

More than $45 \%$ of respondents reported using their cuff at least weekly, and nearly two-thirds reported using their cuff at least monthly. Less than one-third reported never using their cuff (Figure 3). Of participants using their cuff less than a few times per week, $58.5 \%$ stated they would be more likely to take their HBP if they

Figure 2. Demographics of surveyed nonreporters $(n=316)$.

\begin{tabular}{|c|c|c|c|c|}
\hline \multicolumn{5}{|c|}{ Demographics of surveyed non-reporters $(n=316)$} \\
\hline Age* & \multicolumn{4}{|c|}{ Mean: 61.4 years \pm 13.3 years } \\
\hline Gender* & \multicolumn{2}{|l|}{$35.1 \%$ Male } & \multicolumn{2}{|l|}{$64.9 \%$ Female } \\
\hline Practice Location & \multicolumn{2}{|l|}{$65.8 \%$ Rural } & \multicolumn{2}{|l|}{$34.2 \%$ Urban } \\
\hline Preferred Language & \multicolumn{2}{|l|}{ 85.8\% English } & \multicolumn{2}{|l|}{$14.2 \%$ Spanish } \\
\hline Race & $52.2 \%$ White & $3.8 \%$ Black & $3.0 \% \mathrm{~A} / \mathrm{Pl} / \mathrm{NA}^{\dagger}$ & $42.0 \%$ Other/Unspecified \\
\hline Diabetic & \multicolumn{2}{|l|}{$32.3 \%$ Yes } & \multicolumn{2}{|l|}{$67.7 \%$ No } \\
\hline Reported Previously & \multicolumn{2}{|l|}{$37.7 \%$ Yes } & \multicolumn{2}{|l|}{$62.3 \%$ No } \\
\hline \multicolumn{5}{|c|}{$\begin{array}{l}\text { *Significant differences were seen with age and gender demographics between survey } \\
\text { participants and non-reporters who did not participate in the survey }(p<0.0001 \text { and } p=0.0211 \\
\text { respectively) } \\
+\mathrm{A} / \mathrm{PI} / \mathrm{NA} \text { is Asain/Pacific Islander/Native American }\end{array}$} \\
\hline
\end{tabular}


Figure 3. Cuff usage among nonreporters.

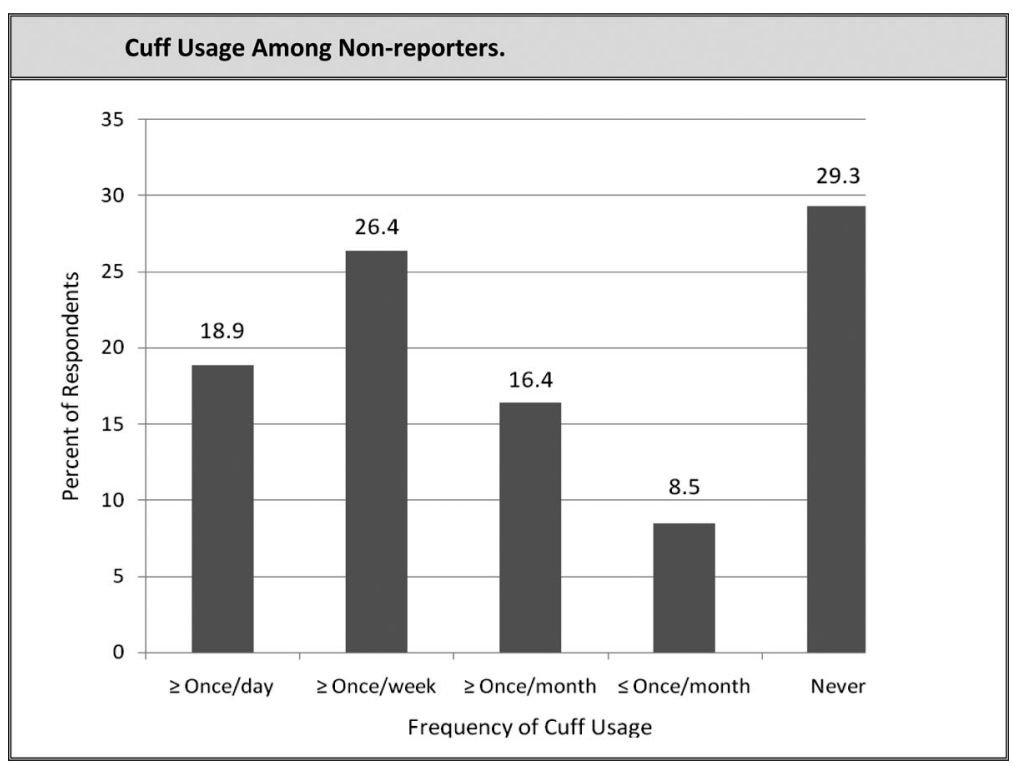

were told they could take it less often than the program's recommendation to check it daily. In the multivariate logistic regression analysis, the only significant factor associated with cuff usage was language (Figure 4). Spanish speakers were 4.4 times more likely to be nonusers of cuffs (95\% CI, 2.22-8.885). Age, sex, having diabetes, clinic location (rural versus urban), and previous

Figure 4. Odds ratios of cuff usage among nonreporters. ${ }^{17}$

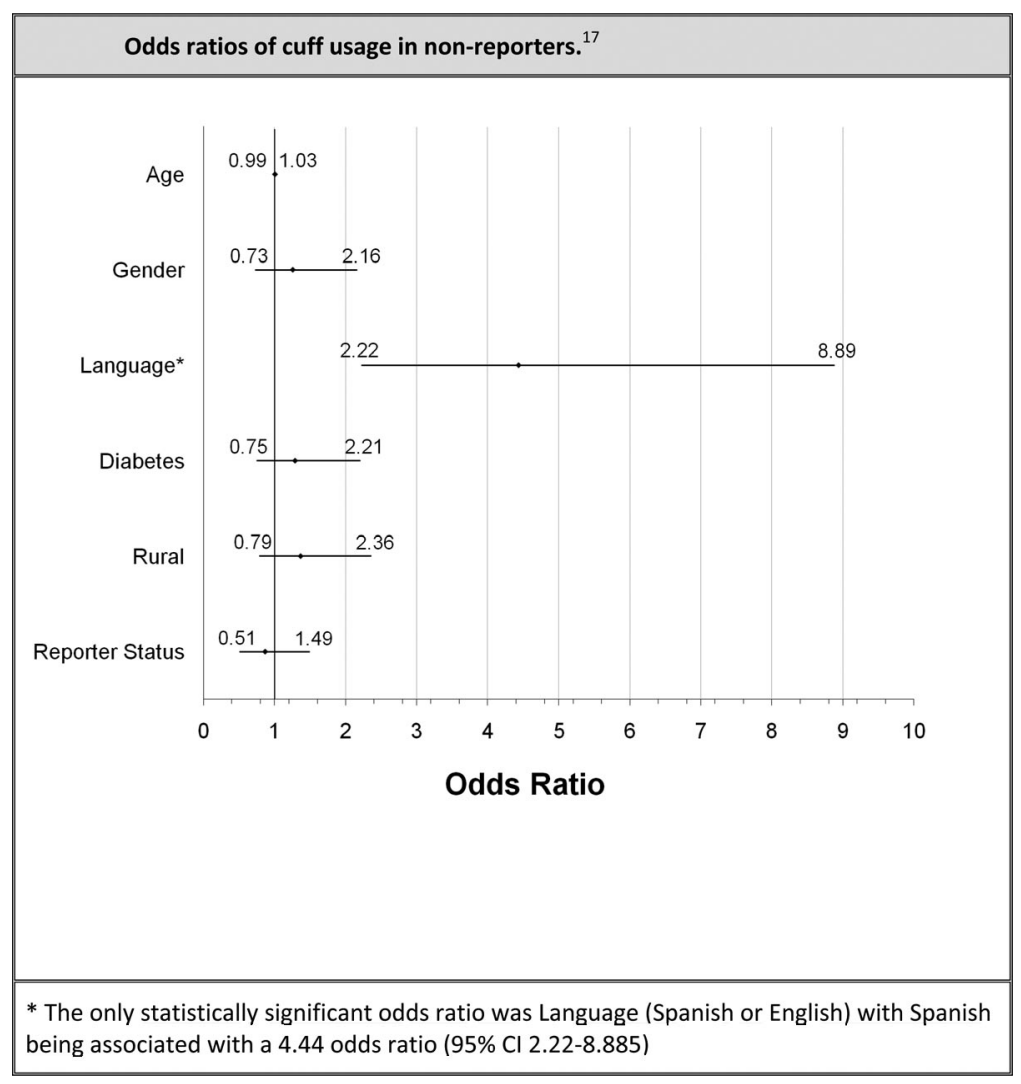


use of the reporting system were not significant predictors of cuff usage. Common reasons for not using the HBP cuff given during the qualitative interviews were forgetting, having normal BP, lack of time, concern about accuracy, thinking office readings were superior, self-described laziness, and the cuff not working.

Among participants using their cuff during the past few months, the majority (58\%) reported sharing readings with providers independent of the central reporting system. Despite using their cuffs, the remaining $42 \%$ did not share their HBP values with their providers. Open-ended questioning explored barriers to sharing readings with providers, using HBP cuffs, and reporting via the automated system (Figure 5). Whereas personal factors were most commonly cited as reasons for not using the HBP cuff and formal reporting system, clinic factors were most commonly cited for not sharing HBP values with providers. Common reasons for not sharing HBP readings included not having a doctor visit/appointment, the provider did not ask about HBP, and the patient's belief that office-measured BP was sufficient/superior. General BP knowledge or program knowledge was the second largest contributor to all aspects of nonparticipation. Example responses of personal, clinic, and knowledge factors also are shown in Figure 5. Interestingly, anxiety was not one of the common personal factors described; only 5 of 213 respondents (2.3\%) specifically listed anxiety as a reason for not using their cuff regularly. Selected responses to qualitative questions are shown in Figure 6.

The third aim of this evaluation was to increase participation through motivational patient education. This was accomplished during interviews by answering patient questions about cuff usage and the reporting system, as well as reinforcing the importance of HBPM and reporting. Impact was measured by studying reporting habits after the survey was conducted. More than 2 years after the survey, more than 3 times more surveyed nonreporters $(24.4 \%)$ began using the central reporting system compared with nonreporters who were not surveyed $(7.5 \% ; P<.0001)$.

\section{Discussion}

This is the first in-depth investigation of why patients do not initiate or continue participation

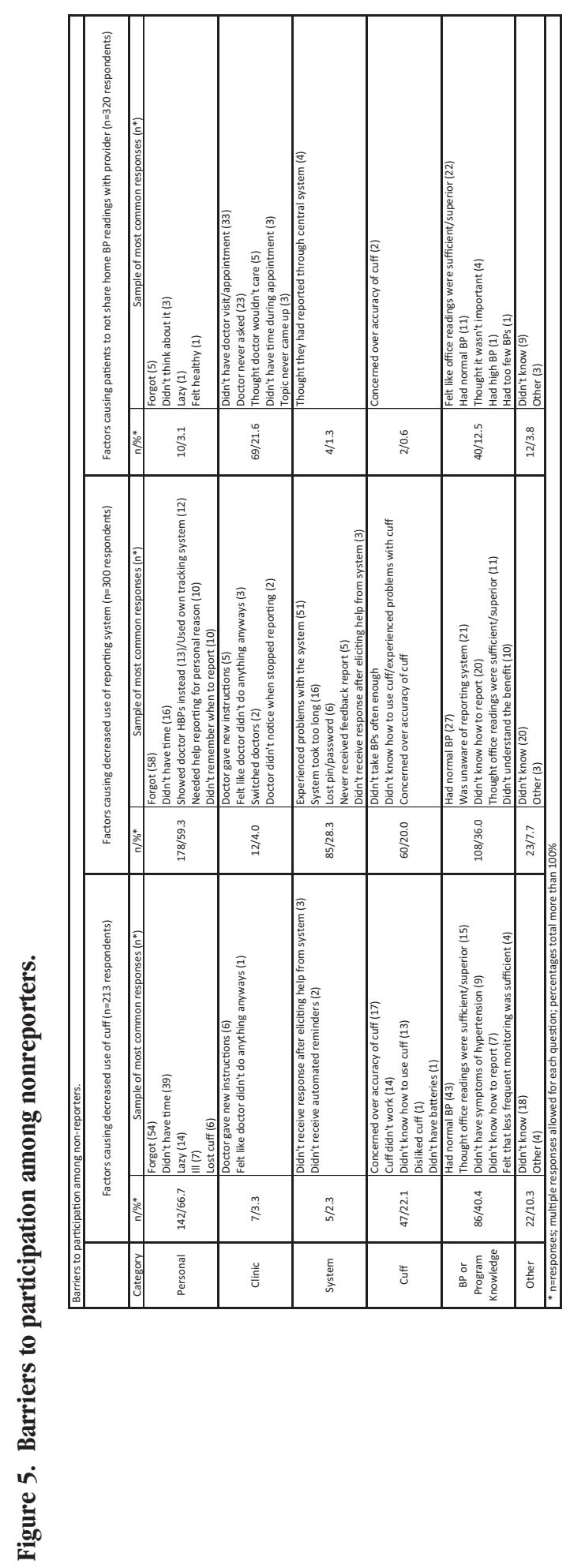


Figure 6. Sample responses to open ended questions.

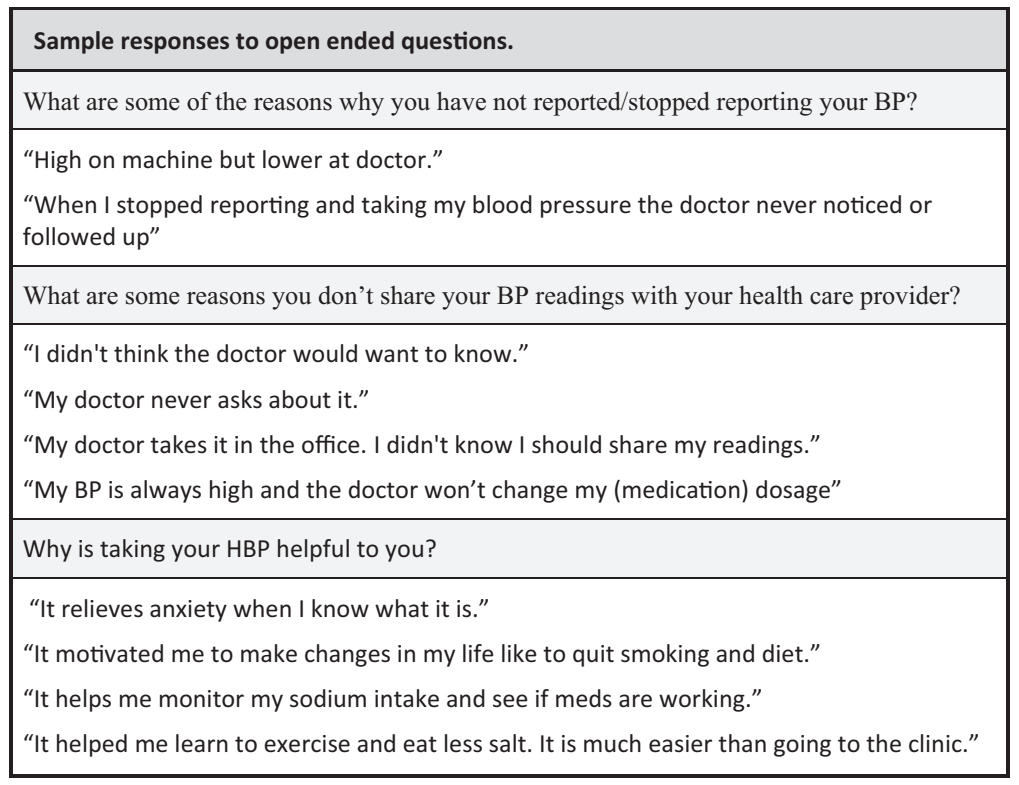

with a HBP reporting system. We found that the majority of nonreporters (70.2\%) still used their HBP cuffs despite not reporting through the central system. This frequency is greater than that found in a previous cross-sectional study of hypertensives at primary care clinics, where $43 \%$ were currently using HBPM. ${ }^{9}$ In our investigation, a large majority of patients who still used their cuffs were also communicating their HBP values to their health care provider directly (58\%). However, it is concerning that $42 \%$ of HBP users were not sharing their readings with providers; this leaves a large amount of valuable clinical information unutilized for treatment goals. Some patients described not sharing HBP results with their doctor because home values were higher than office values. Providers may have missed opportunities to identify and treat uncontrolled or masked hypertension, which has been associated with increased cardiovascular risk. $^{18,19}$

Reasons why patients who monitored their HBP did not communicate their readings to providers were largely attributable to clinic factors, suggesting clinicians and clinic systems have potential areas for improvement. Because HBPM is becoming more common and many patients did not share results if their provider did not ask, asking patients if they monitor HBPs should become standard practice. Clinics could consider adding a space for
HBP next to office BP in the vitals section of a chart, and encourage staff who are assessing vitals to inquire at that time. Increasing awareness of HBPM and educating patients about its usefulness may increase the flow of this valuable clinical information.

Reasons for not monitoring HBPs were largely personal factors. Previous literature, including the AHA's Call to Action statement, cited anxiety as an important personal factor involved in HBPM and listed it as a contraindication to HBPM in certain patients. ${ }^{11}$ A previous qualitative study with 13 subjects concluded that the fear of increasing anxiety through HBPM may be overemphasized. ${ }^{20}$ Our evaluation did not find anxiety to be a major contributor to nonparticipation, suggesting that the interaction between HBPM and anxiety may be overstated.

A previous study that implemented HBPM for 1 year found that only $50 \%$ of patients were willing to continue HBPM after the trial ended. A lack of motivation was not considered to be a major barrier based on their analysis. ${ }^{13}$ In contrast, our study found the most prevalent personal factors affecting nonusage of $\mathrm{HBP}$ monitors were forgetting, not having time, and self-described laziness. This suggests HBPM was not a lifestyle priority to subjects, and a lack of motivation does contribute to nonparticipation in HBPM. Lack of knowledge about both blood pressure and the program design were 
also common causes of nonparticipation. Many patients did not understand the benefit of home monitoring and expressed confusion about the variability in readings. Some personal factors and lack of education may be modifiable. Providers may achieve increased participation through education, motivational interview techniques, and adherence strategies.

After being surveyed, A CARE nonparticipants were more likely to increase their use of the central reporting system than A CARE nonparticipants who were not surveyed. This suggests that providers can increase participation through follow-up. Automated reminder systems and reminders during clinic visits should be implemented with nonparticipants.

This evaluation found that among nonreporters, Spanish speakers were much less likely to be using their cuffs. This adds to the body of literature documenting ethnic disparities in hypertension. ${ }^{2,5,6}$ The A CARE program was designed as a bilingual program in English and Spanish, with instructional videos, booklets, and the telephone and internet systems available in both languages. Provider and clinic language competency was not measured, and this could cause a language barrier despite the pro- gram's bilingual design. In addition, possible confounding variables not measured include cultural competency, literacy, and socioeconomic status. These factors, in addition to language barriers, are all well-documented for causing health disparities. ${ }^{14,21-23}$

Previous studies have cited cost as a potential barrier to home blood pressure monitoring. We did not evaluate the relative importance of this barrier because home blood pressure monitors were provided to each patient at no cost.

In this study, many patients stated they would be more likely to monitor HBP if they could be expected to do so less frequently. After the initiation of this HBPM program, the AHA suggested a different schedule for obtaining accurate HBP. Recommendations included more frequent monitoring during an initial phase and then deceased monitoring for a long-term observation phase after achieving control of BP. ${ }^{11}$ This program did not include a feature to decrease monitoring frequency after BP was controlled. Future HBPM programs could suggest different monitoring schedules or decreasing monitoring frequency for patients with controlled hypertension. However, because this study found that lack of knowl-

Figure 7. Summary recommendations for improving patient participation in home blood pressure monitoring programs.

\begin{tabular}{|l}
\hline $\begin{array}{l}\text { Summary Recommendations for Improving Patient Participation in Home Blood } \\
\text { Pressure Monitoring Programs }\end{array}$ \\
\hline $\begin{array}{l}\text { 1. Ask all patients about home blood pressure results and discuss at every hypertension } \\
\text { follow up visit }\end{array}$ \\
\hline 2. Add home blood pressure to routinely collected office vital sign data \\
3. Provide enhanced patient education explaining: \\
- Why blood pressure control matters \\
- Hole of home versus office blood pressure readings \\
- Accuracy of home blood pressure monitoring \\
- Lack of symptoms with high blood pressure \\
\hline $\begin{array}{l}\text { 4. Provide suggestions/materials to help patients start a home blood pressure monitoring } \\
\text { routine }\end{array}$ \\
\hline 5isits. Decrease amount of monitoring for patients with controlled hypertension \\
individual patients
\end{tabular}


edge about program design also contributed to nonparticipation, it is important that the home monitoring regimen be simple; a less frequent but complex regimen may also prove to be a barrier to participation.

This study may be useful for maximizing participation in future HBPM programs. A summary of recommendations is shown in Figure 7. Programs should focus on patient motivation and education to optimize impact. Design should minimize time involvement and include a reminder system. All providers, regardless of participation in a formalized HBPM program, should inquire about HBPM because some patients monitor HBP but do not readily share this information unless asked.

\section{Limitations}

Respondents were limited to those with telephone numbers and who answer telephones. However, contact numbers were collected from clinic databases, in which many patients listed private cell phones and work phones, minimizing bias compared with telephone surveys that use phone books listing home numbers. In addition, morning, afternoon, and evening calls were made in an attempt to minimize sampling bias.

Sampling methodologies were designed to maximize the quality improvement aim of our study and thus were not based strictly on a random probability sampling approach. The sample for this study over-represented patients from small clinics and patients with uncontrolled hypertension. It is possible that clinic factors may be different in smaller clinics, but it is unlikely that personal factors or knowledge of the program would differ by clinic size. The survey sample was older and had more women compared with other nonreporters. Although statistically significant, the absolute difference in mean age was 4 years, and there is no reason to believe this small age difference or female predominance would significantly bias results. Finally, patients may have been reluctant to disclose that they were not following providers' instructions and hesitant to offer critical remarks about clinic and provider factors.

During the qualitative analysis, patient responses were grouped into broader categories, but there was overlap between these categories. For example, even though "forgetting to take HBP" was considered a personal factor, clinic factors and system factors may have also contributed to this response if the automated reminder system was ineffective or providers did not follow-up with inactive participants. When identifying potential system improvements, improvements in one category can alleviate deficiencies in other categories.

\section{Conclusion}

In this investigation of nonparticipation in an HBPM program, the large majority of respondents were still using their HBP cuffs despite not using the formal reporting system. This finding is encouraging for this project and other self-management programs; it suggests most "dropouts" may still be receiving clinical benefit from the intervention. Furthermore, contacting patients about nonparticipation may increase involvement. Understanding nonparticipation in this HBPM program may be generalizable for self-management programs in other disciplines that experience difficulties with retention.

The authors thank Lori Crane, $\mathrm{PhD}$, for her analysis of methodology for this publication; Allison Hall for her work in data gathering and data analysis; and Elizabeth Staton, MSTC, for her work with manuscript revisions and technical writing.

\section{References}

1. Chobanian AV, Bakris GL, Black HR, et al. The Seventh Report of the Joint National Committee on Prevention, Detection, Evaluation, and Treatment of High Blood Pressure: the JNC 7 report. JAMA 2003;289(19):2560-72.

2. Egan BM, Zhao Y, Axon RN. US trends in prevalence, awareness, treatment, and control of hypertension, 1988-2008. JAMA 2010;303(20):2043-50.

3. Kannel WB. Prevalence and implications of uncontrolled systolic hypertension. Drugs Aging 2003;20(4):277-86.

4. Haroun MK, Jaar BG, Hoffman SC, Comstock GW, Klag MJ, Coresh J. Risk factors for chronic kidney disease: a prospective study of 23,534 men and women in Washington County, Maryland. J Am Soc Nephrol 2003;14(11):2934-41.

5. Ong KL, Cheung BM, Man YB, Lau CP, Lam KS. Prevalence, awareness, treatment, and control of hypertension among United States adults 1999-2004. Hypertension 2007;49(1):69-75.

6. Multi-Sponser Surveys, Inc. The 2006 Gallup Study of the hypertension market. Princeton, NJ: MultiSponser Surveys, Inc.; 2006.

7. Little P, Barnett J, Barnsley L, Marjoram J, FitzgeraldBarron A, Mant D. Comparison of acceptability of and preferences for different methods of measuring blood pressure in primary care. BMJ 2002; 325(7358):258-9. 
8. Viera AJ, Cohen LW, Mitchell CM, Sloane PD. Use of home blood pressure monitoring by hypertensive patients in primary care: survey of a practice-based research network cohort. J Clin Hypertens (Greenwich) 2008;10(4):280-6.

9. Viera AJ, Cohen LW, Mitchell CM, Sloane PD. How and why do patients use home blood pressure monitors? Blood Press Monit 2008;13(3):133-7.

10. Poon IO, Etti N, Lal LS. Does the use of home blood pressure monitoring vary by race, education, and income? Ethn Dis 2010;20(1):2-6.

11. Pickering TG, Miller NH, Ogedegbe G, Krakoff LR, Artinian NT, Goff D. Call to action on use and reimbursement for home blood pressure monitoring: a joint scientific statement from the American Heart Association, American Society Of Hypertension, and Preventive Cardiovascular Nurses Association. Hypertension 2008;52(1):10-29.

12. Cappuccio FP, Kerry SM, Forbes L, Donald A. Blood pressure control by home monitoring: meta-analysis of randomised trials. BMJ 2004;329(7458):145.

13. Port K, Palm K, Viigimaa M. Daily usage and efficiency of remote home monitoring in hypertensive patients over a one-year period. J Telemed Telecare 2005;11(Suppl 1):34-6.

14. Thoolen B, de Ridder D, Bensing J, Gorter K, Rutten G. Who participates in diabetes self-management interventions? Issues of recruitment and retainment. Diabetes Educ 2007;33(3):465-74.

15. Gazmararian JA, Ziemer DC, Barnes C. Perception of barriers to self-care management among diabetic patients. Diabetes Educ 2009;35(5):778-88.

16. Verberk WJ, Kroon AA, Kessels AG, de Leeuw PW. Home blood pressure measurement: a systematic review. J Am Coll Cardiol 2005;46(5):743-51.

17. Clark O, Djulbegovic B. Forest plots in excel software. Data sheet. 2001.

18. Pierdomenico SD, Cuccurullo F. Prognostic value of white-coat and masked hypertension diagnosed by ambulatory monitoring in initially untreated subjects: an updated meta analysis. Am J Hypertens 2011;24:52-8.

19. Ogedegbe G, Agyemang C, Ravenell JE. Masked hypertension: evidence of the need to treat. Curr Hypertens Rep 2010;12(5):349-55.

20. Rickerby J, Woodward J. Patients' experiences and opinions of home blood pressure measurement. J Hum Hypertens 2003;17(7):495-503.
21. Ishikawa H, Yano E, Fujimori S, et al. Patient health literacy and patient-physician information exchange during a visit. Fam Pract 2009;26(6):517-23.

22. Williams MV, Baker DW, Parker RM, Nurss JR. Relationship of functional health literacy to patients' knowledge of their chronic disease. A study of patients with hypertension and diabetes. Arch Intern Med 1998;158(2):166-72.

23. Carbone ET, Rosal MC, Torres MI, Goins KV, Bermudez OI. Diabetes self-management: perspectives of Latino patients and their health care providers. Patient Educ Couns 2007;66(2):202-10.

24. Asmar R, Zanchetti A. Guidelines for the use of selfblood pressure monitoring: a summary report of the First International Consensus Conference. Group Evaluation \& Measure of the French Society of Hypertension. J Hypertens 2000;18(5):493-508.

25. Fields LE, Burt VL, Cutler JA, Hughes J, Roccella EJ, Sorlie P. The burden of adult hypertension in the United States 1999 to 2000: a rising tide. Hypertension 2004;44(4):398-404.

26. Green BB, Cook AJ, Ralston JD, et al. Effectiveness of home blood pressure monitoring, Web communication, and pharmacist care on hypertension control: a randomized controlled trial. JAMA 2008; 299(24):2857-67.

27. Klem ML, Wing RR, McGuire MT, Seagle HM, Hill JO. A descriptive study of individuals successful at long-term maintenance of substantial weight loss. Am J Clin Nutr 1997;66(2):239-46.

28. Lorig KR, Sobel DS, Stewart AL, et al. Evidence suggesting that a chronic disease self-management program can improve health status while reducing hospitalization: a randomized trial. Med Care 1999;37(1):5-14.

29. Mazze RS, Simonson GD, Robinson RL, et al. Characterizing blood pressure control in individuals with type 2 diabetes: the relationship between clinic and self-monitored blood pressure. Diabet Med 2003;20(9):752-7.

30. Ostchega Y, Yoon SS, Hughes J, Louis T. Hypertension awareness, treatment, and control-continued disparities in adults: United States, 2005-2006. NCHS Data Brief 2008;(3):1-8.

31. Pickering T. Recommendations for the use of home (self) and ambulatory blood pressure monitoring. American Society of Hypertension Ad Hoc Panel. Am J Hypertens 1996;9(1):1-11. 